\title{
Differences in history of sexual behavior between patients with oropharyngeal squamous cell carcinoma and patients with squamous cell carcinoma at other head and neck sites
}

\author{
Kristina R. Dahlstrom, MS ${ }^{1}$, Guojun Li, MD, $\mathrm{PhD}^{1,2}$, Guillermo Tortolero-Luna, MD, $\mathrm{PhD}^{3}$, \\ Qingyi Wei, MD, PhD ${ }^{2}$, and Erich M. Sturgis, MD, MPH ${ }^{1,2}$ \\ ${ }^{1}$ Department of Head and Neck Surgery, The University of Texas M. D. Anderson Cancer \\ Center, Houston, Texas \\ 2 Department of Epidemiology, The University of Texas M. D. Anderson Cancer Center, Houston, \\ Texas \\ 3 Department of Cancer Control and Population Sciences, University of Puerto Rico \\ Comprehensive Cancer Center, San Juan, Puerto Rico
}

\begin{abstract}
Background-An emerging epidemic of human papillomavirus (HPV)-associated oropharyngeal cancer has been proposed. We compared the sexual behaviors of patients with squamous cell carcinoma of the oropharynx (SCCOP) and patients with squamous cell carcinoma of non-oropharyngeal head and neck sites (SCCNOP) to expand our understanding of sexual behavior as a risk factor for HPV-associated head and neck cancer.
\end{abstract}

Methods-The sexual history of 165 SCCOP patients and 87 SCCNOP patients was determined in a hospital-based case-case comparison study.

Results-SCCOP patients were significantly more likely than SCCNOP patients to have had $>9$ lifetime sex partners (odds ratio $[\mathrm{OR}]=39.2 ; 95 \%$ confidence interval $[\mathrm{CI}]=8.2-187.3$ ), to have engaged in oral-genital sex $(\mathrm{OR}=3.5 ; 95 \% \mathrm{CI}=1.1-11.1)$, and to have had $>4$ oral-genital sex partners $(\mathrm{OR}=8.6 ; 95 \% \mathrm{CI}=2.2-33.4)$.

Conclusions-The findings of this study suggest that some risk factors are site specific and provide further evidence that certain sexual behaviors increase the risk of HPV-associated SCCOP.

\section{Keywords}

head and neck neoplasms; human papillomavirus; sexual behavior; oropharynx; HPV16

\section{INTRODUCTION}

\begin{abstract}
Squamous cell carcinomas (SCCs) arising in the upper aerodigestive tract (i.e., the oral cavity, oropharynx, hypopharynx, and larynx) are strongly associated with cigarette smoking. In the United States, incidences of SCC of the oral cavity, hypopharynx, and larynx have been falling for two decades, most likely as a result of the drop in cigarette smoking prevalence over the past four decades. ${ }^{1,2}$ However, the incidence of oropharyngeal cancer (SCCOP) has been rising approximately $5 \%$ per year for a decade. ${ }^{2-4}$ Historically,
\end{abstract}

Corresponding Author: Erich M. Sturgis, MD, MPH, Unit 441, 1515 Holcombe Blvd., Houston, TX 77030; Tel: 713-792-5432; Fax: 713-794-4662; esturgis@mdanderson.org. 
the overwhelming majority of SCCs of the head and neck (including those of the oropharynx) have occurred in the sixth and seventh decades of life and have been strongly associated with tobacco and alcohol exposures. Recently, however, the proportion of these cancers - chiefly those of the oropharynx-occurring in young to middle-aged adults and in people with limited tobacco and alcohol exposures has been increasing. ${ }^{2-5}$ In the past decade, many patients with SCCOP have been found to be seropositive for oncogenic human papillomavirus (HPV) (up to 60\% of patients) and/or to have oncogenic HPV DNA identified in their tumors (up to $80 \%$ and overwhelmingly HPV16); in contrast, patients with nonoropharyngeal head and neck SCCs (SCCNOP) have much lower HPV seroprevalence and HPV DNA tumor prevalence (typically $<20 \%$ for both). ${ }^{4,6-11}$

Oncogenic HPV is well established as the main risk factor for cervical cancer and is understood to be sexually transmitted. ${ }^{12,13}$ However, while the mode of transmission of oncogenic HPV to the oropharynx is likely also sexual, the data supporting an oral-genital mode of transmission are limited. ${ }^{14-16}$ Other studies have explored the association between sexual behavior and oncogenic HPV-associated head and neck cancer, but no effort has been made to carefully compare sexual behaviors between patients with tumors at different head and neck cancer sites. $., 10,17$ Oncogenic HPV is associated specifically with SCCOP, and this failure to segregate analyses by tumor site has the potential to result in underestimation of the risk of SCCOP associated with sexual behaviors. ${ }^{6,10,14,17}$ Furthermore, much of the current literature is limited to reports on series of 100 or fewer patients with SCCOP with limited detail about specific risk behaviors. $6,10,17$

We conducted a hospital-based case-case study to compare the sexual behavior characteristics of patients with SCCOP and patients with SCCNOP (oral cavity, hypopharynx, and larynx). Additionally, in a subgroup of patients with available HPV16 serologic data, we compared the prevalence of certain sexual behaviors between seropositive and seronegative patients. The rising proportion of head and neck cancers in the oropharynx, 18 the rising incidence of such cancers in young and middle-aged adults, ${ }^{5}$ and the declining fraction of cases attributable to cigarette smoking ${ }^{2}$ all indicate the importance of establishing which behaviors other than tobacco smoking may be responsible for the changing demographics of head and neck cancer. Determining the behavioral risk factors for head and neck cancer is imperative for future cancer prevention efforts and for understanding the potential chemopreventive role of HPV vaccines. ${ }^{19,20}$ This study expands our understanding of the role of sexual behavior as a risk factor for SCCOP and as a marker of HPV16 exposure.

\section{MATERIALS AND METHODS}

\section{Participants}

All living patients who had been prospectively enrolled in a molecular epidemiology study conducted at The University of Texas M. D. Anderson Cancer Center between May 1996 and August 2006 were invited to participate. Participants in that study (and the current study) met the following criteria: newly diagnosed, previously untreated SCC of the head and neck (oral cavity, oropharynx, hypopharynx, or larynx); resident of the United States; at least 18 years old at diagnosis. Patients with prevalent cancers (excluding nonmelanoma skin carcinomas) and patients with incident cancers of the lip, nasopharynx, nose/paranasal sinuses, or neck (unknown primary site) were excluded. This study was approved by the institutional review boards of both M. D. Anderson Cancer Center and The University of Texas School of Public Health. 


\section{Data Collection Procedures}

Patients who agreed to participate (after being contacted by phone) were mailed an institutional review board-approved informed consent form and a sexual history questionnaire with separate postage-paid return envelopes. The questionnaire included no personal identifiers except a unique confidential research code. Participants were asked about lifetime sexual practices and history of sexually transmitted diseases before the cancer diagnosis.

In addition to information from the sexual history questionnaire, we analyzed information that had been prospectively collected from patients at the time of enrollment in the parent molecular epidemiology study. For this parent study, patients were recruited upon presentation to M. D. Anderson with an incident SCC of the head and neck, and at enrollment, they completed a standardized self-administered questionnaire with questions about sociodemographic information as well as alcohol and tobacco exposures. Subjects were considered ever-smokers if they had smoked at least 100 cigarettes in their lifetime and ever-drinkers if they had consumed alcoholic beverages at least once a week for a year or more during their lifetime. Subjects who had previously smoked or consumed alcoholic beverages but had not done so in the year prior to their cancer diagnosis were considered former smokers and former drinkers, respectively. Furthermore, at enrollment in the parent molecular epidemiologic study, participants provided a blood sample for laboratory analysis, which included HPV16 serology in a subset of patients. For the parent study, virus-like particles generated from recombinant baculovirus-infected insect cells were used to detect antibodies against HPV16 in the plasma of study subjects using a standard ELISA as described previously. 8,21

\section{Data Analysis}

The study population was described using standard descriptive statistics. Differences in demographic and tobacco and alcohol exposures between case groups were compared using Pearson's chi-square or Fisher's exact test for categorical variables and Student's $t$-test for continuous variables with Satterthwaite's adjustment for unequal variances where indicated. Categorical variables were created to describe each study subject's demographic, socioeconomic, and clinical characteristics and smoking and alcohol exposures. For the analysis comparing sexual behavior, categorical variables were created on the basis of median values for the entire study population. Logistic regression models were used to estimate odds ratios [ORs] and 95\% confidence intervals [CIs] for the association between sexual behavior and head and neck cancer site (oropharynx versus other sites) as well as for the association between HPV16 seropositivity versus HPV16 seronegativity. The backward stepwise selection procedure was used to identify confounders from among the demographic, socioeconomic, and exposure variables using $P<.2$ as the cutoff. $P<.05$ was used to determine statistical significance, and all tests were 2-sided. Analyses were performed using Stata 9.0 (Stata Corporation, College Station, Texas).

\section{RESULTS}

Of the 1,270 patients who participated in the prospective molecular epidemiologic study, $831(65 \%)$ were alive and were mailed the sexual history questionnaire. Of these, 252 returned completed questionnaires and signed informed consent forms (30\% response rate). Responders were significantly more likely to be non-Hispanic white, have higher income and education, be never smokers or former smokers, have poorly differentiated tumors, higher-stage tumors, and tumors located in the oropharynx than nonresponders (data not shown). Eighty-seven (21\%) of 419 SCCNOP patients and 165 (40\%) of 412 SCCOP patients returned questionnaires. Both SCCNOP and SCCOP responders were more likely to 
have higher education and income, while SCCNOP responders were also more likely to be white and female than nonresponders (data not shown).

\section{Demographic, Socioeconomic, and Exposure Characteristics and Tumor Sites}

The demographic, socioeconomic, and exposure characteristics of SCCNOP and SCCOP patients are compared in Table 1. SCCOP patients were younger (mean 53.6, median 53) than SCCNOP patients (mean 57.1, median 59; $P=.02$ ) and were also more likely to be male, non-Hispanic white, have higher income and education, and be a never smoker (Table 1). HPV 16 serologic data were available on 46 SCCOP patients (23 positive, 50\%) and 32 SCCNOP patients (4 positive, $12.5 \% ; P<.01$ ).

\section{Sexual Behaviors and Exposures}

Sexual behavior and exposure information is shown in Table 2. The average age at first sexual intercourse was 17.1 years (median 17) for SCCOP patients and 17.5 years (median $17)$ for SCCNOP patients $(P=.24)$. SCCOP patients had on average more lifetime sex partners and there was a trend of increasing risk of SCCOP with increasing number of lifetime sex partners, even after multivariate adjustment $\left(P_{\text {trend }}<.01\right)$. The mean number of lifetime sex partners was 37.7 (median 11) for SCCOP patients and 9.7 (median 4.5) for SCCNOP patients $(P=.07)$.

SCCOP patients were significantly more likely to have ever engaged in oral-genital sex, with 55\% having had more than 4 such partners over their lifetime compared to only $18 \%$ for SCCNOP patients. Additionally, there was a trend of increasing risk of oropharyngeal site with increasing number of lifetime oral-genital sex partners, even after multivariable adjustment $\left(P_{\text {trend }}<.01\right)$. The mean number of oral-genital sex partners was 25.9 (median 6) for SCCOP patients and 4.5 (median 1$)$ for SCCNOP patients $(P=.13)$. SCCOP patients were more likely to have had oral-anal sex and to have more lifetime oral-anal sex partners than SCCNOP patients, but these differences were not statistically significant. Nor was there any evidence of a significant trend of increasing risk of oropharyngeal site associated with increasing number of oral-anal sex partners $\left(P_{\text {trend }}=.26\right)$. Finally, SCCOP patients were more likely than SCCNOP patients to have had a partner with a history of an abnormal Pap test, although this was not significant after multivariable adjustment.

\section{Relevant Medical History}

Table 3 presents the relevant medical history information obtained from the sexual history questionnaire. We found no evidence that prior tonsillectomy had any protective effect. Reporting a history of sexually transmitted disease was significantly more common among SCCOP than SCCNOP patients, but these differences were not statistically significant after multivariable adjustment. No patient had a history of HIV infection. Each reported sexually transmitted disease was more frequent in SCCOP patients than in SCCNOP patients, although the difference was significant only for gonorrhea (the difference approached significance for genital warts). Among female patients, $43 \%$ of those with SCCOP had a history of an abnormal Pap test, compared with only $19 \%$ of those with SCCNOP, but this difference was not significant after multivariable adjustment. Lesions of viral etiology not considered sexually transmitted (cold sores and non-genital warts) were both more common among SCCOP patients than among SCCNOP patients.

\section{Patient Characteristics and Sexual Exposures by HPV16 Status}

HPV16 serology data were available for $78(31 \%)$ of the 252 patients who responded to the survey. Of these patients, two-thirds $(\mathrm{n}=51)$ were seronegative and one-third $(\mathrm{n}=27)$ were seropositive. There were no significant differences between the two groups in the 
demographic, socioeconomic, and exposure variables examined (data not shown). There was a higher proportion of males in the HPV16 seropositive group, but this difference was not statistically significant $(P=.08)$. As expected, the cancers in the HPV16-seropositive patients were overwhelmingly ( $89 \%$ ) oropharyngeal cancers, while the cancers in the HPV16seronegative patients were relatively evenly split between the oral cavity and the oropharynx (41\% and $45 \%$, respectively).

Sexual behaviors and exposure information obtained from the sexual history questionnaire for patients with known HPV16 status are shown in Table 4. Because of the limited sample size $(n=78)$, we chose to present only dichotomized data. The HPV16-seropositive group tended to have a higher proportion of patients in the high-risk sexual behavior categories for all questions, but a significant difference was observed only for oral-genital sex (Table 4). All HPV16-seropositive patients reported having had oral-genital sex and when lifetime number of oral-genital sex partners was dichotomized, patients reporting 2 or more lifetime partners were more likely to be HPV16-seropositive, although this was not statistically significant after multivariable adjustment.

\section{DISCUSSION}

Oncogenic HPV is well established as the main risk factor for cervical cancer and has more recently received attention as an etiological agent for some head and neck cancers (primarily SCCOP). The results of our study suggest that certain sexual behaviors are associated with a subset of head and neck cancers, specifically tumors arising in the oropharynx. The main behaviors associated with SCCOP were having a large number of lifetime sex partners, engaging in oral-genital contact, and having a large number of oral-genital sex partners. These findings remained significant after adjustment for demographic, socioeconomic, and exposure characteristics.

We found that an increasing number of lifetime sex partners was associated with an increasing risk of oropharyngeal cancer site and that having had more than 9 lifetime sex partners increased the risk of oropharyngeal cancer site 34-fold. These findings are consistent with those of others, who have previously found a positive association between lifetime number of sex partners and risk of SCC of the head and neck. ${ }^{6,10,17}$ For example, D'Souza et al found an association between a high lifetime number of vaginal-sex partners ( 26 or more) and oropharyngeal cancer $(\mathrm{OR}=3.1 ; 95 \% \mathrm{CI}=1.5-6.5) .{ }^{6} \mathrm{Smith}$ et al found that in a group of patients with oral cavity and oropharynx SCC, younger patients ( $\leq 55$ years) who reported 4 or more lifetime sex partners were 4.4 times as likely to have high-risk HPV types detected in their tumor as were older patients ( $>55$ years) with 3 or fewer lifetime sex partners. ${ }^{17}$ Furthermore, Schwartz et al found that oral cavity and oropharynx SCC patients with 15 or more lifetime sex partners were 2.5 times as likely to have HPV16-positive tumors as were patients with less than 15 partners. ${ }^{10}$

While HPV16 serologic status is a marker for sexual behavior in general, ${ }^{22,} 23$ we would assume that the most likely mode of transmission of HPV16 to the upper aerodigestive tract is through oral-genital contact. In this study, we found significant associations between SCCOP and oral-genital sex and lifetime number of oral-genital sex partners. There was a dose-response effect of rising risk of oropharyngeal cancer site with rising number of oralgenital sex partners; SCCOP patients were 16 times as likely as SCCNOP patients to have had more than 4 lifetime partners. Previous studies have also found an association between engaging in oral-genital sex and having an HPV-positive oral cavity or oropharyngeal tumor. ${ }^{6,} 17,24$ In a case-control study, cases were more than 3 times as likely as controls to have had 6 or more lifetime oral-genital sex partners; when the analysis was restricted to HPV16-positive patients, cases were almost 9 times as likely to have had 6 or more lifetime 
oral-genital sex partners. ${ }^{6}$ Ritchie et al found in a study of patients with SCC of the oral cavity and oropharynx that the prevalence of oral-genital sex was 4.2-fold higher (95\% CI=1.5-11.7) among patients with HPV-positive SCC than among patients with HPVnegative SCC. ${ }^{24}$ Smith et al, found that the odds of having engaged in oral-genital contact were 4.8-times higher among oral cavity and oropharyngeal cancer patients 55 years or younger than among patients older than 55 years $(95 \% \mathrm{CI}=1.9-12.1) .{ }^{17}$ Recently, D'Souza et al evaluated the association between oral sexual behaviors and HPV infection and found that the odds of oral HPV infection increased significantly with the number of oral-genital sex and open-mouth kissing partners among two control groups. ${ }^{14}$ Taken together, these findings and our own findings support oral-genital sex as a major mode of transmission of oncogenic HPV to the upper aerodigestive tract.

SCCOP patients were more likely to have had a partner with a history of an abnormal Pap test but not with having had a partner with cervical cancer and neither association remained significant in regression analyses. The possible association between SCCOP and having had a partner with a history of an abnormal Pap test is in accordance with a previous report of increased incidence of tonsillar and tongue cancer among husbands of women with cervical cancer. ${ }^{25} \mathrm{We}$ also found that women with SCCOP were more likely than women with SCCNOP to have had an abnormal Pap test themselves prior to their cancer diagnosis. This finding was of only borderline statistical significance and was not significant in regression analyses; however, the small sample size may have contributed to this lack of statistical significance. Large population-based studies using SEER data and other cancer-registry data have confirmed that women with a history of cervical cancer have an increased risk of HPVassociated second primary cancers, ${ }^{26-28}$ and this association is especially strong for SCCOP. ${ }^{26,}{ }^{29}$ The increased risk of HPV-associated second primary cancers after a diagnosis of an HPV-associated primary cancer appears to hold true for men as well. ${ }^{30}$

Analysis of history of sexually transmitted diseases revealed that both a history of gonorrhea and any sexually transmitted disease were significantly more common among SCCOP patients; however, only a history of gonorrhea remained significantly associated with SCCOP in regression analyses. Several studies have found HPV seropositivity and subsequently the risk of cervical cancer to be increased among women with history of gonorrhea, herpes simplex virus- 2 or $C$. trachomatis. ${ }^{31-34}$ Consequently, it seems plausible that coinfections with bacterial or viral pathogens modulate the risk of HPV-associated SCC, possibly by modulating host immune function, increasing susceptibility to HPV, or delaying viral clearance.

Our study had several limitations. First, the low response rate may bias the results if differences exist between the responders and nonresponders with respect to sexual history. The response rate was $40 \%$ among the SCCOP patients but only $21 \%$ among the SCCNOP patients. Furthermore, SCCOP patients were more likely to have higher education and income than the SCCNOP patients. However, the SCCNOP patients who did respond tended to have higher education and income than those who did not respond. This indicates that the SCCNOP responders were more similar to the SCCOP responders than were the SCCNOP patients overall and therefore may also be more similar with respect to sexual behavior. This being the case, the association between sexual behavior and SCCOP may be underestimated in our study. Furthermore, the low response rate resulted in a small sample size, which limited statistical power and our ability to perform any meaningful subgroup analyses.

Second, the retrospective nature of this study creates a recall bias. While we would expect such recall bias to be nondifferential, several factors might suggest otherwise. For instance, members of the group with SCCOP were younger on average and might have had better memory. Additionally, the age difference could have introduced a cohort effect such that 
between-group differences in the prevalence of sexual behaviors reflect the years during which patients were born more than differences in tumor site. It would have been convenient to have the two groups be more similar with regard to the demographic, socioeconomic, and exposure characteristics or to have our sample size be large enough to permit rigorous subgroup analyses; however, we did include these variables in our analyses, and our principal findings were relatively stable after adjustment for them.

Third, nondifferential misclassification may have occurred due to the subject matter of the survey and the willingness of the responders to answer sensitive questions. Moreover, differential misclassification could also have occurred owing to the demographic and clinical differences between the SCCOP and SCCNOP patients. A large number of responders failed to completely fill out the questionnaire, resulting in missing data; furthermore, some may have underreported certain behaviors because of associated societal stigmas. Both of these factors would be likely to bias the effect estimate toward the null; however, the anonymous nature of the survey may have mitigated some of the underreporting bias.

Fourth, we used serology to ascertain HPV status. The estimated sensitivity of this technique is $50-75 \%$, indicating a number of possible false-negative results, which would lead to misclassification of HPV16-positive patients as HPV16-negative. This could lead to an underestimation of the association between sexual behavior and HPV16 serologic status. Additionally, serological HPV status may not reflect an infection in the oropharynx, but rather could be due to an infection isolated to the anogenital region.

Fifth, we grouped patients by cancer site because HPV is primarily associated with the oropharynx; however, we can assume that some SCCOP patients were HPV16-negative and some SCCNOP patients were HPV16-positive. For the 42 patients for whom we had HPV16 tumor DNA status available, $19 \%$ of SCCOP patients were actually HPV16-negative while $44 \%$ of SCCNOP patients were HPV16-positive. Unfortunately, since we only had this data available for a small minority of patients $(17 \%)$ we were unable to compare by HPV tumor status. Regardless, because our site-specific results may be diluted by HPV negative tumors in the SCCOP group it is likely that there is an underestimation of sexual behaviors associated with HPV-associated cancer. We are currently assessing tumor HPV16 and HPV18 DNA status for all prospectively recruited patients and will include this data in future studies.

While head and neck cancers have traditionally been associated with primarily tobacco and alcohol use it appears that the risk factor profile is changing for these cancers, and an accurate understanding of risk factors is critical to establish effective prevention strategies. We conclude that among patients with SCC of the head and neck, having numerous sex partners (in particular oral-genital sex partners) is a risk factor for oropharyngeal cancer site. We feel that these findings add to the evidence that HPV16 can be sexually transmitted to the upper aerodigestive tract and suggest that oral-genital sex is a risk factor for HPV16associated SCCOP. A number of models exploring the impact of the HPV16/18 vaccine currently targeted at girls and young women predict a marked decrease in the incidence of cervical cancer over the next several decades. ${ }^{35,} 36$ Unfortunately, the overwhelming majority of HPV16-associated SCCOP occur in males, and the current vaccination strategy, which targets girls and young women, will benefit men only secondarily as vaccinated women age and the incidence of chronic oncogenic HPV infection in sexually active females declines. Such an effect could take a generation to achieve. We encourage the rapid study of the efficacy, safety, and cost-effectiveness of HPV16/18 vaccines in males and, if results warrant, the vaccination of young adult and adolescent males. 


\section{Acknowledgments}

Funding/Support: Supported by an NIH SPORE grant in head and neck cancer Career Development Award (to E.M.S., awardee and Waun Ki Hong, principal investigator); NIH P-30 grant CA-16672 (to The University of Texas M.D. Anderson Cancer Center); NIH R01 grant CA-131274 (to Q.W.); NIH R01 grant ES-11740 (to Q.W.); NIH grant R03 CA128110-01A1 (to E.M.S.); NIH R03 grant CA-135679 (to G.L.); NIH K07 grant CA-133099 (to G.L.); NIH K-12 grant 88084 (to E.M.S., faculty trainee and R.C. Bast, principal investigator); The University of Texas M. D. Anderson Cancer Center Institutional Research Grants (to E. M.S.); and M. D. Anderson Cancer Center start-up funds (to E.M.S.)

\section{References}

1. Jemal A, Thun MJ, Ries LA, et al. Annual report to the nation on the status of cancer, 1975-2005, featuring trends in lung cancer, tobacco use, and tobacco control. J Natl Cancer Inst. 2008; 100(23): 1672-1694. [PubMed: 19033571]

2. Sturgis EM, Cinciripini PM. Trends in head and neck cancer incidence in relation to smoking prevalence: An emerging epidemic of human papillomavirus-associated cancers? Cancer. 2007; 110(7):1429-1435. [PubMed: 17724670]

3. Chaturvedi AK, Engels EA, Anderson WF, Gillison ML. Incidence trends for human papillomavirus-related and -unrelated oral squamous cell carcinomas in the united states. J Clin Oncol. 2008; 26(4):612-619. [PubMed: 18235120]

4. Ernster JA, Sciotto CG, O'Brien MM, et al. Rising incidence of oropharyngeal cancer and the role of oncogenic human papilloma virus. Laryngoscope. 2007; 117(12):2115-2128. [PubMed: 17891052]

5. Shiboski CH, Schmidt BL, Jordan RC. Tongue and tonsil carcinoma: Increasing trends in the U.S. population ages 20-44 years. Cancer. 2005; 103(9):1843-1849. [PubMed: 15772957]

6. D’Souza G, Kreimer AR, Viscidi R, et al. Case-control study of human papillomavirus and oropharyngeal cancer. N Engl J Med. 2007; 356(19):1944-1956. [PubMed: 17494927]

7. Kreimer AR, Clifford GM, Boyle P, Franceschi S. Human papillomavirus types in head and neck squamous cell carcinomas worldwide: A systematic review. Cancer Epidemiol Biomarkers Prev. 2005; 14(2):467-475. [PubMed: 15734974]

8. Dahlstrom KR, Adler-Storthz K, Etzel CJ, et al. Human papillomavirus type 16 infection and squamous cell carcinoma of the head and neck in never-smokers: A matched pair analysis. Clin Cancer Res. 2003; 9(7):2620-2626. [PubMed: 12855639]

9. Mork J, Lie AK, Glattre E, et al. Human papillomavirus infection as a risk factor for squamous-cell carcinoma of the head and neck. N Engl J Med. 2001; 344(15):1125-1131. [PubMed: 11297703]

10. Schwartz SM, Daling JR, Doody DR, et al. Oral cancer risk in relation to sexual history and evidence of human papillomavirus infection. J Natl Cancer Inst. 1998; 90(21):1626-1636. [PubMed: 9811312]

11. Gillison ML, Koch WM, Capone RB, et al. Evidence for a causal association between human papillomavirus and a subset of head and neck cancers. J Natl Cancer Inst. 2000; 92(9):709-720. [PubMed: 10793107]

12. Baseman JG, Koutsky LA. The epidemiology of human papillomavirus infections. J Clin Virol. 2005; 32 (Suppl 1):S16-24. [PubMed: 15753008]

13. Dillner J, Andersson-Ellstrom A, Hagmar B, Schiller J. High risk genital papillomavirus infections are not spread vertically. Rev Med Virol. 1999; 9(1):23-29. [PubMed: 10371669]

14. D’Souza G, Agrawal Y, Halpern J, Bodison S, Gillison ML. Oral sexual behaviors associated with prevalent oral human papillomavirus infection. J Infect Dis. 2009; 199(9):1263-1269. [PubMed: 19320589]

15. Kreimer AR, Alberg AJ, Daniel R, et al. Oral human papillomavirus infection in adults is associated with sexual behavior and HIV serostatus. J Infect Dis. 2004; 189(4):686-698. [PubMed: 14767823]

16. Kreimer AR, Alberg AJ, Viscidi R, Gillison ML. Gender differences in sexual biomarkers and behaviors associated with human papillomavirus-16, -18 , and -33 seroprevalence. Sex Transm Dis. 2004; 31(4):247-256. [PubMed: 15028941] 
17. Smith EM, Ritchie JM, Summersgill KF, et al. Age, sexual behavior and human papillomavirus infection in oral cavity and oropharyngeal cancers. Int J Cancer. 2004; 108(5):766-772. [PubMed: 14696105]

18. Carvalho AL, Nishimoto IN, Califano JA, Kowalski LP. Trends in incidence and prognosis for head and neck cancer in the united states: A site-specific analysis of the SEER database. Int J Cancer. 2005; 114(5):806-816. [PubMed: 15609302]

19. Shaw AR. Human papillomavirus vaccines in development: If they're successful in clinical trials, how will they be implemented? Gynecol Oncol. 2005; 99(3 Suppl 1):S246-8. [PubMed: 16213009]

20. Devaraj K, Gillison ML, Wu TC. Development of HPV vaccines for HPV-associated head and neck squamous cell carcinoma. Crit Rev Oral Biol Med. 2003; 14(5):345-362. [PubMed: 14530303]

21. Kirnbauer R, Hubbert NL, Wheeler CM, Becker TM, Lowy DR, Schiller JT. A virus-like particle enzyme-linked immunosorbent assay detects serum antibodies in a majority of women infected with human papillomavirus type 16. J Natl Cancer Inst. 1994; 86(7):494-499. [PubMed: 8133532]

22. Dillner J, Kallings I, Brihmer C, et al. Seropositivities to human papillomavirus types 16, 18, or 33 capsids and to chlamydia trachomatis are markers of sexual behavior. J Infect Dis. 1996; 173(6): 1394-1398. [PubMed: 8648211]

23. Olsen AO, Dillner J, Gjoen K, Magnus P. Seropositivity against HPV 16 capsids: A better marker of past sexual behaviour than presence of HPV DNA. Genitourin Med. 1997; 73(2):131-135. [PubMed: 9215097]

24. Ritchie JM, Smith EM, Summersgill KF, et al. Human papillomavirus infection as a prognostic factor in carcinomas of the oral cavity and oropharynx. Int J Cancer. 2003; 104(3):336-344. [PubMed: 12569557]

25. Hemminki K, Dong C, Frisch M. Tonsillar and other upper aerodigestive tract cancers among cervical cancer patients and their husbands. Eur J Cancer Prev. 2000; 9(6):433-437. [PubMed: 11201683]

26. Chaturvedi AK, Engels EA, Gilbert ES, et al. Second cancers among 104,760 survivors of cervical cancer: Evaluation of long-term risk. J Natl Cancer Inst. 2007; 99(21):1634-1643. [PubMed: 17971527]

27. Balamurugan A, Ahmed F, Saraiya M, et al. Potential role of human papillomavirus in the development of subsequent primary in situ and invasive cancers among cervical cancer survivors. Cancer. 2008; 113(10 Suppl):2919-2925. [PubMed: 18980275]

28. Chaturvedi AK, Kleinerman RA, Hildesheim A, et al. Second cancers after squamous cell carcinoma and adenocarcinoma of the cervix. J Clin Oncol. 2009; 27(6):967-973. [PubMed: 19114696]

29. Rose Ragin CC, Taioli E. Second primary head and neck tumor risk in patients with cervical cancer--SEER data analysis. Head Neck. 2008; 30(1):58-66. [PubMed: 17657793]

30. Sikora AG, Morris LG, Sturgis EM. Bidirectional association of anogenital and oral cavity/ pharyngeal carcinomas in men. Arch Otolaryngol Head Neck Surg. 2009; 135(4):402-405. [PubMed: 19380365]

31. Silins I, Kallings I, Dillner J. Correlates of the spread of human papillomavirus infection. Cancer Epidemiol Biomarkers Prev. 2000; 9(9):953-959. [PubMed: 11008914]

32. Kjaer SK, Dahl C, Engholm G, Bock JE, Lynge E, Jensen OM. Case-control study of risk factors for cervical neoplasia in denmark. II. role of sexual activity, reproductive factors, and venereal infections. Cancer Causes Control. 1992; 3(4):339-348. [PubMed: 1617121]

33. Stone KM, Zaidi A, Rosero-Bixby L, et al. Sexual behavior, sexually transmitted diseases, and risk of cervical cancer. Epidemiology. 1995; 6(4):409-414. [PubMed: 7548350]

34. Smith JS, Herrero R, Bosetti C, et al. Herpes simplex virus-2 as a human papillomavirus cofactor in the etiology of invasive cervical cancer. J Natl Cancer Inst. 2002; 94(21):1604-1613. [PubMed: 12419786]

35. Barnabas RV, Laukkanen P, Koskela P, Kontula O, Lehtinen M, Garnett GP. Epidemiology of HPV 16 and cervical cancer in finland and the potential impact of vaccination: Mathematical modelling analyses. PLoS Med. 2006; 3(5):e138. [PubMed: 16573364] 
36. Goldie SJ, Grima D, Kohli M, Wright TC, Weinstein M, Franco E. A comprehensive natural history model of HPV infection and cervical cancer to estimate the clinical impact of a prophylactic HPV-16/18 vaccine. Int J Cancer. 2003; 106(6):896-904. [PubMed: 12918067] 
Table 1

Demographics, socioeconomics, exposures, and cancer subsites of SCCOP and SCCNOP patients

\begin{tabular}{|c|c|c|c|}
\hline & Nonoropharynx $(n=87)$ & Oropharynx $(n=165)$ & \\
\hline & No. $(\%)$ & No. $(\%)$ & $P$-Value \\
\hline Age in years & & & .01 \\
\hline$<40$ & $6(7.0)$ & $11(6.7)$ & \\
\hline $40-49$ & 13(14.9) & $41(24.9)$ & \\
\hline $50-59$ & $27(31.0)$ & $72(43.6)$ & \\
\hline $60-69$ & $33(37.9)$ & $35(21.2)$ & \\
\hline$>69$ & $8(9.2)$ & $6(3.6)$ & \\
\hline Sex & & & $<.01$ \\
\hline Male & $51(58.6)$ & $144(87.3)$ & \\
\hline Female & $36(41.4)$ & 21(12.7) & \\
\hline Ethnicity & & & $.02^{*}$ \\
\hline Non-Hispanic White & $78(89.7)$ & $160(97.0)$ & \\
\hline Other & $9(10.3)$ & $5(3.0)$ & \\
\hline Income & $\mathrm{n}=84$ & $\mathrm{n}=159$ & $<.01$ \\
\hline Less than $\$ 25,000$ & $13(15.5)$ & $10(6.3)$ & \\
\hline$\$ 25,000-\$ 74,999$ & $42(50.0)$ & $55(34.6)$ & \\
\hline Over $\$ 74,999$ & $29(34.5)$ & $94(59.1)$ & \\
\hline Education & $\mathrm{n}=87$ & $\mathrm{n}=163$ & .01 \\
\hline High school graduate/GED or less & $32(36.8)$ & $30(18.4)$ & \\
\hline Tech/assoc degree/some college & $24(27.6)$ & $53(32.5)$ & \\
\hline Bachelor's or advanced degree & $31(35.6)$ & $80(49.1)$ & \\
\hline Smoking status & & & $<.01$ \\
\hline Never & $18(20.7)$ & $64(38.8)$ & \\
\hline Former & $33(37.9)$ & $73(44.2)$ & \\
\hline Current & $36(41.4)$ & $28(17.0)$ & \\
\hline Drinking status & & & .29 \\
\hline Never & $27(31.0)$ & $40(24.2)$ & \\
\hline Former & $20(23.0)$ & $32(19.4)$ & \\
\hline Current & $40(46.0)$ & $93(56.4)$ & \\
\hline \multicolumn{4}{|l|}{ Cancer site } \\
\hline Oropharynx & & $165(100.0)$ & \\
\hline Oral cavity & $48(55.2)$ & & \\
\hline Hypopharynx & $7(8.0)$ & & \\
\hline Larynx & $32(36.8)$ & & \\
\hline
\end{tabular}




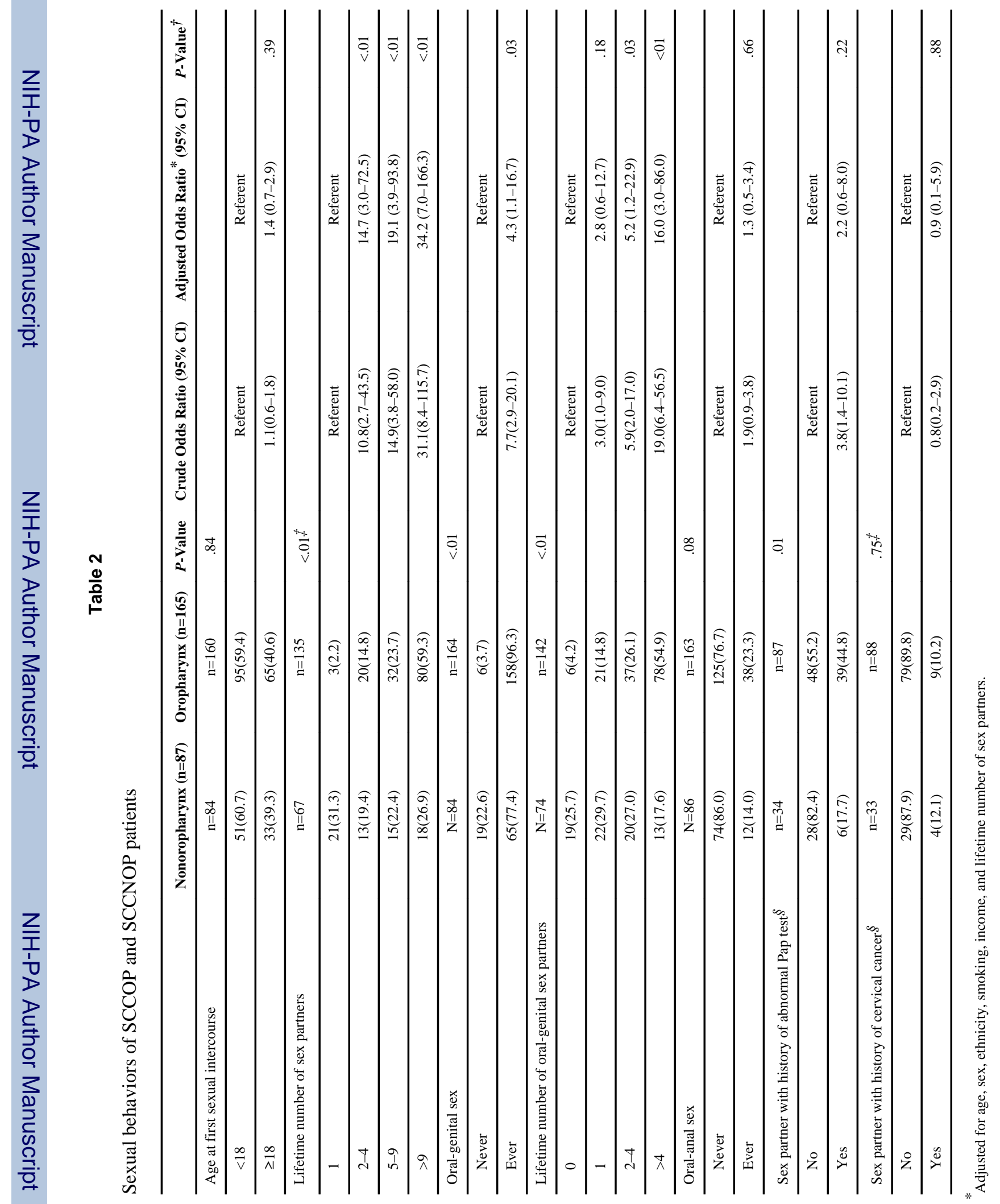




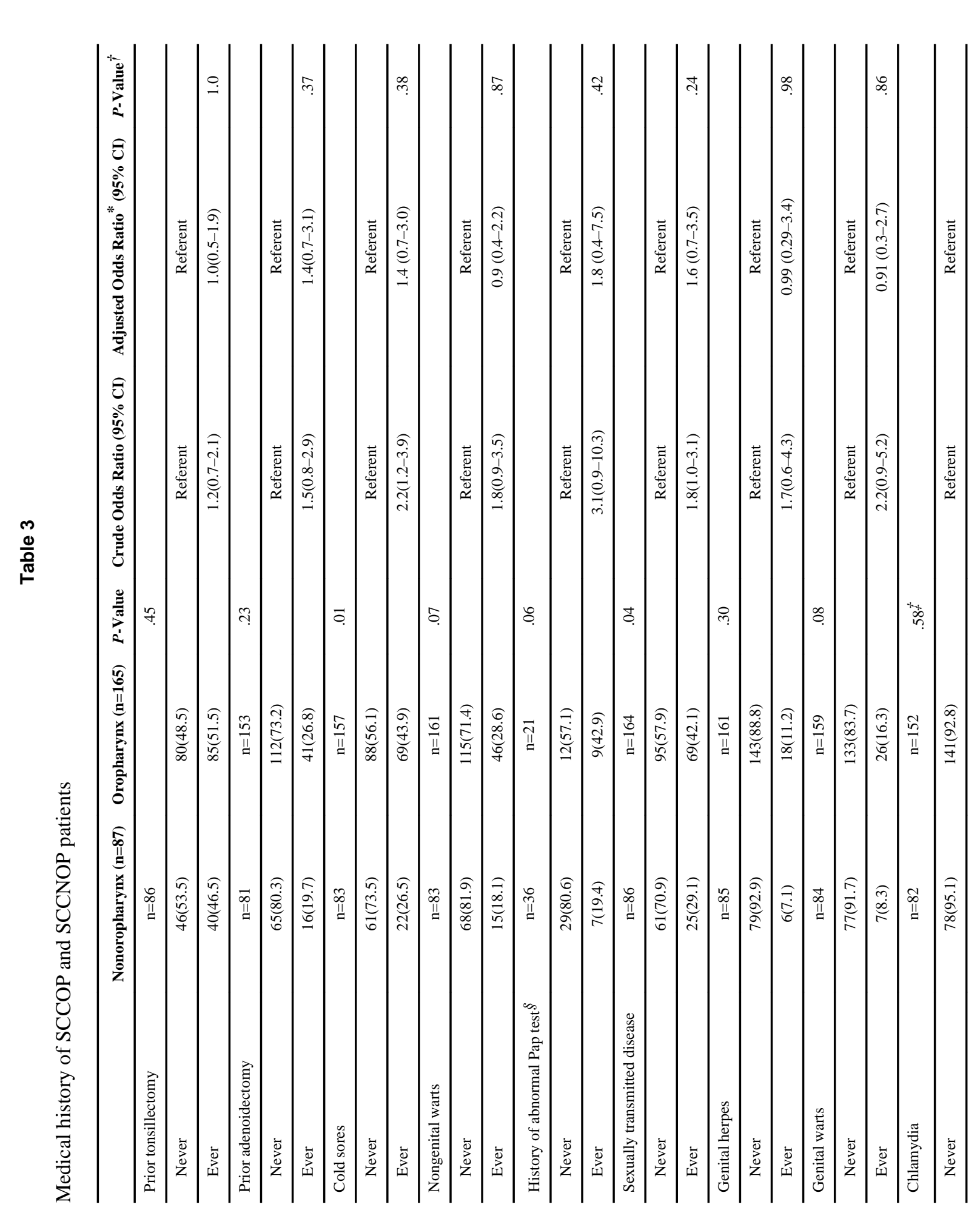


Dahlstrom et al.

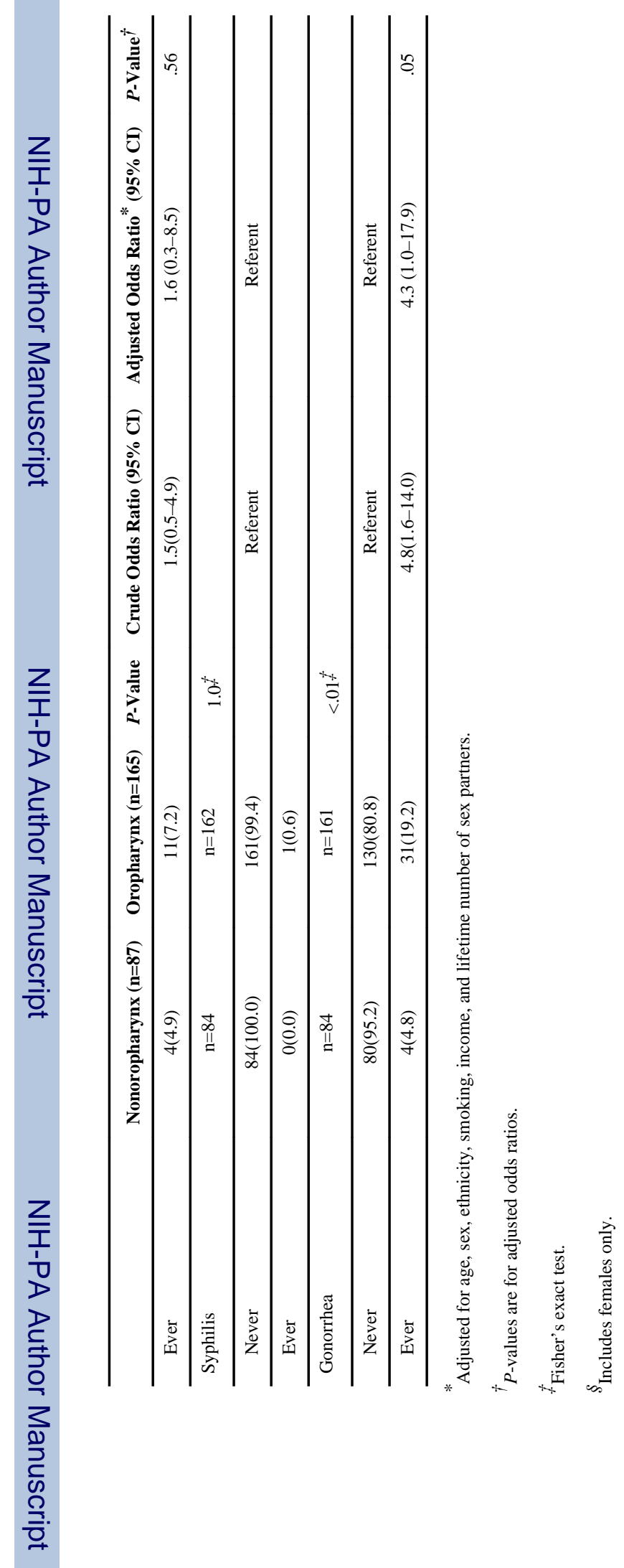

Head Neck. Author manuscript; available in PMC 2012 June 1. 


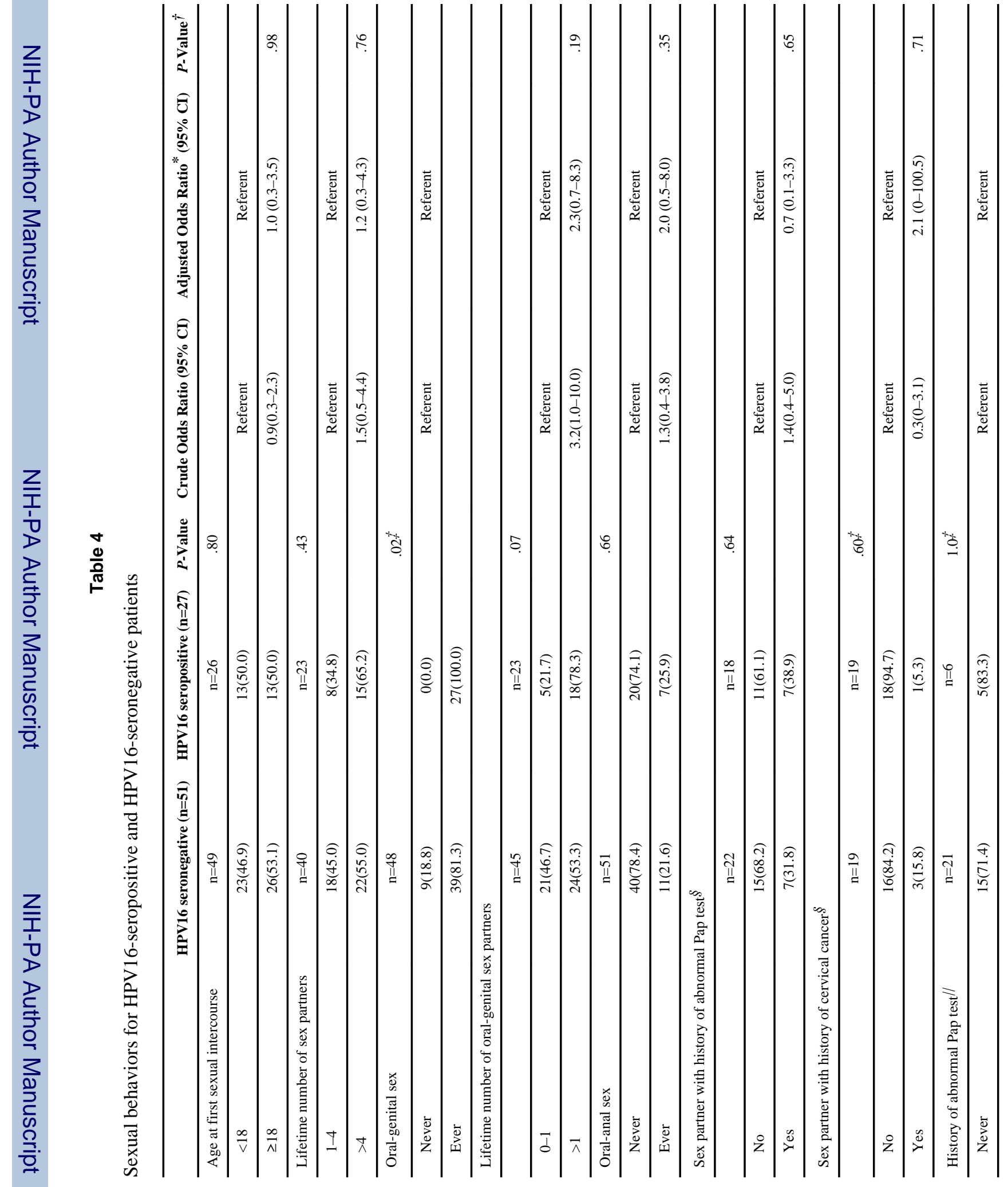




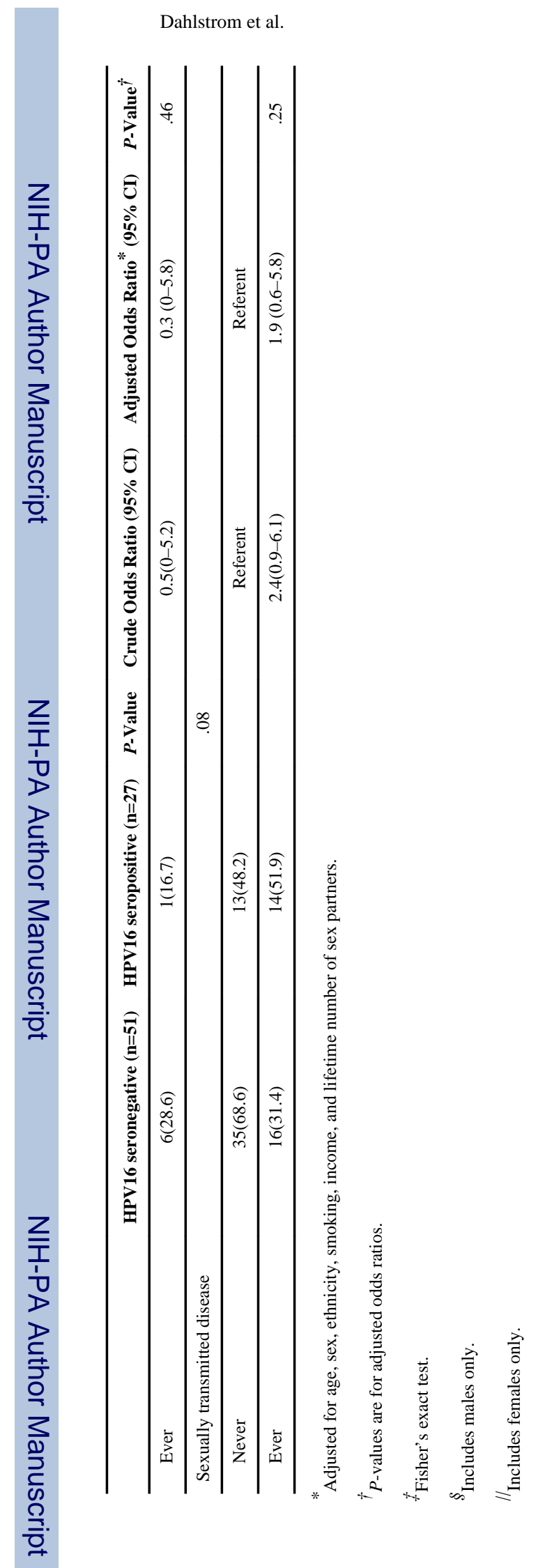

Head Neck. Author manuscript; available in PMC 2012 June 1. 